\title{
«Nous sommes tous des migrants»
}

Jean Martin

jean.martin[at]saez.ch
Le Docteur Jean-Claude Métraux est un pédopsychiatre vaudois qui s'est impliqué de manière principale, depuis les années 1980, dans les soins et la relation avec des personnes aux cadres de référence culturels et sociaux différents des nôtres, ceci à l'étranger ou en Suisse. Il a co-fondé en 1993 l'association Appartenances et a été son directeur durant huit ans (les associations Appartenances de Genève et Lausanne ont publié en 2009 un substantiel ouvrage sur leur expérience [1]). Il enseigne à la Faculté des sciences sociales et politiques de Lausanne et participe à de multiples activités de formation.

Après un premier livre [2], décrivant le «deuil congelé» chez des personnes ayant vécu des circonstances particulièrement traumatiques (deuil qui ne peut se «dégeler» que plus tard, quand les conditions l'autorisent), il publie un nouvel ouvrage: «La migration comme métaphore» [3]. Substantiel, étayé par son expérience (de nombreux exemples sont décrits) et une importante bibliographie francophone. Présentant sa trajectoire et le bilan qu'il tire aujourd'hui de ce qui est fait dans nos sociétés, particulièrement en Suisse, dans nos rapports avec les migrants. Avec une tonalité critique, plutôt sombre à mon sens: nous n'avons pas fait juste, nous sommes restés sous l'emprise de modèles ethno-centrés, jugeants, discriminatoires, au mieux paternalistes.

\section{Un récit attachant, I'histoire du narrateur}

Dans la première partie «Nous sommes tous des migrants» Métraux parle de lui, de son enfance, de ses parents et grands-parents qui ont émigré de la campagne à la ville; d'un grand-oncle très engagé, à qui il a fait plusieurs visites en Allemagne de l'Est. Jeune pédopsychiatre, il se passionne pour la révolution sandiniste du Nicaragua, où il vit de 1987 à 1990 - et où, non sans un certain malaise, il se voit immédiatement considéré comme un expert. Puis il crée Appartenances à Lausanne, ce qui nous a amené à de nombreux contacts professionnels, dans le cadre de ma fonction de médecin cantonal, et amicaux. Il vit une année à Sarajevo (2001-2002).

Cette partie inclut une phénoménologie de la migration: ce que veut dire quitter un monde, entrer dans un monde nouveau puis y vivre, puis en être; toutes choses différentes et successives. Discutant les notions d'intégration (idéalement créatrice) vs assimilation, marginalisation, ghettoïsation.
Point important à garder à l'esprit, les sédentaires aussi connaissent des expériences du registre de la migration: changement de lieu ou de travail, chômage, prise de retraite, changement de statut (divorce, veuvage), ou même situations d'addiction ou de délinquance.

\section{Les attitudes et pratiques prévalentes - une analyse sévère}

Dans son introduction, l'auteur annonce la couleur: «Nous peinons avec l'altérité. Pire, penchés depuis des lustres sur les maux des naufragés [les migrants], nous tendons à creuser l'abîme qui nous sépare. Chercheurs et praticiens, nous piétinons, enchevêtrés dans nos nœuds de certitudes. Ce livre propose une perspective alternative.» Un peu plus loin: «Nous élargissons d'une main la fracture sociale que l'autre tente de combler, propageons l'épidémie que nous déclarons vouloir soigner (...) Décortiqués, placés sous la loupe de l'interculturalité, les migrants souffrent chaque jour davantage de notre négligence du lien entre aidants et aidés.»

C'est dans la deuxième partie, «Relents d'orientalisme» (référence à une vision occidentale supérieure par rapport aux faits exotiques), qu'il argumente sa charge à l'endroit de nos manières de faire jusqu'ici. Il juge que professionnels comme chercheurs adhèrent encore beaucoup trop à des modèles où les déficits (des migrants) et les discriminations jouent un rôle indû; évoque des «accointances de la stratégie [fédérale] Migration et santé avec les pouvoirs politique, économique et académique», de professionnels complices («malgré eux?» sic). A son sens, le modèle dit des déficits reprend de la vigueur alors qu'il s'était

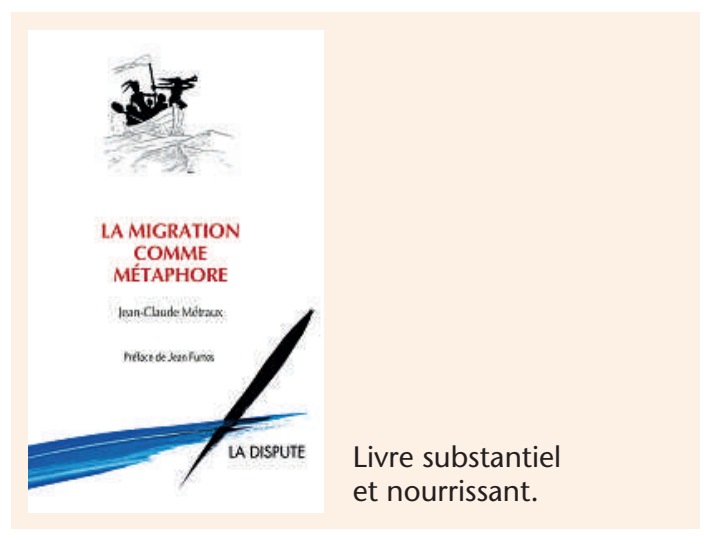




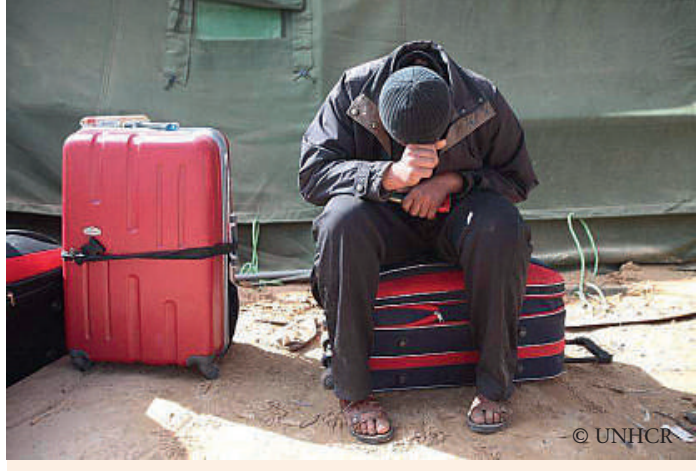

La migration, une expérience traumatique qui demande un accueil et des soins compétents, «co-construisant» avec la personne dans un esprit de reconnaissance mutuelle.

essoufflé il y a une vingtaine d'années. Il livre une critique des conclusions de plusieurs volets du Programme national de recherche "Migration et relations interculturelles». Chercheurs comme praticiens négligeraient les ressources des migrants eux-mêmes, qui dans les travaux réalisés «tendent à demeurer des êtres passifs, interdits de voix et de parole propres».

\section{Je regrette dans l'ouvrage certaines condamnations} qui sont généralisantes, apparaissent sans appel.

Avec le «danger d'emprisonner la personne souffrant d'exclusion dans les mailles du filet tendu par les détenteurs du pouvoir». Well..., formulations secouantes.

\section{Etablir une relation thérapeutique basée sur la reconnaissance mutuelle et l'échange}

Dans la troisième partie «Paroles précieuses» sont présentées des pistes pour améliorer les pratiques par «une refonte de nos modes usuels d'échanges, de paroles en particulier». Ceci fondé sur une élaboration théorique approfondie, notamment anthropologique, et sur son expérience professionnelle.

Pour échapper à une situation jugée (très) insatisfaisante, Métraux propose: «Dans ma quête d'alternative crédible, j'utilise la migration comme métaphore. Mes rencontres avec des migrants et des rescapés ont allumé une conscience de nos similitudes propre à étourdir notre obsession des différences. Nous sommes tous des migrants. Nous sommes tous semblables avant d'être différents. Découvrir nos ressemblances nous permet de penser et de développer ensemble, de co-construire un monde de sens partagé.»

Cette partie apporte un cadre fort utile en vue d'évaluer et améliorer les pratiques des aidants, qu'il s'agisse des domaines de la santé, du social ou de l'éducation. Pour «doter nos boussoles d'un nouveau nord», il discute les questions anthropologiques fondamentales du don et contre-don (chapitre VI), de l'échange (en trois catégories, ce qui est monnayé, ce qui est précieux, ce qui est sacré) et de la reconnaissance (chapitre VII). Pour lui, la reconnaissance, au double sens d'identification et surtout de mutualité, prime sur les connaissances: il convient de reconnaître pleinement autrui avant de faire appel à nos techniques professionnelles usuelles.

Il évoque à plusieurs reprises l'importance de l'interprétariat pour une relation adéquate avec les personnes migrantes: «Pour le migrant, l'usage de la langue maternelle, fil invisible le reliant à des origines, enlaçant le passé au présent, abrite d'immenses vertus cicatrisantes (...) Mon activité thérapeutique m'obligea à collaborer avec des interprètes qui m'apprirent une part essentielle du métier. En m'enseignant le monde de sens qui habitait la langue et les gestes de mes patients, ils enfoncèrent dans ma tête le clou de la modestie.» Malgré des progrès quant à la mise en place et au financement de services d'interprétariat, il reste beaucoup à faire [4].

\section{Se souvenir que nous vivons dans un monde imparfait}

Livre substantiel, nourrissant. Toutefois, fort de notre amitié, je fais part de mes réserves. On peut émettre des critiques sur ce qui a été et est entrepris en Suisse et dans des pays comparables. Il est intéressant de détailler comme le fait Métraux ce qui dans des travaux de recherche apparaît comme la persistance d'orientations ne correspondant pas à la «co-construction» qu'il souhaite mais plutôt à des attitudes qui voient chez les migrants des déficits voire entérinent des discriminations. On peut regretter que les Suisses et leurs responsables soient trop peu nombreux à appliquer un modèle de relation marqué par la valorisation du potentiel des personnes migrantes, dans un rapport d'équité et de vraie reconnaissance mutuelle. Cependant, à défaut des solutions idéales, on peut vouloir néanmoins quelque chose d'utile qui peut être réalisé hic et nunc; qui sans être parfait soit proche du bon (ou de «le moins mauvais» - la vie nous y oblige souvent). A cet égard, je regrette dans l'ouvrage certaines condamnations qui sont généralisantes, apparaissent sans appel.

Cela étant, j'apprécie la militance de J.-C. Métraux et même une certaine partialité (oui, il faut être carrément aux côtés de migrants et de ceux qui sont défavorisés). Etre militant voire partial peut être utile pour secouer ceux qui sont trop aisément satisfaits. Et j'ai ici une sorte de «conflit d'intérêt»: je me décris comme politiquement «d'extrême centre» et ai œuvré un quart de siècle comme serviteur public (médecin cantonal), très impliqué dans les problèmes de santé publique et les programmes mis en œuvre pour les résoudre, ou au moins les soulager. J'ai vécu qu'on ne peut pas ne pas tenir compte du contexte, du monde imparfait dans lequel nous vivons. 
Je n'ai jamais été un thuriféraire $d u \ll Y$ en a point comme nous». Mais ce qui a été entrepris depuis une vingtaine d'années dans ce pays (et le canton de Vaud), y compris par Appartenances, n'est pas négligeable du tout et est allé dans le bon sens, voire a des aspects exemplaires. Ainsi en matière de VIH/sida, avec des efforts significatifs en direction des personnes migrantes et allophones, efforts qui ne peuvent pas être catalogués de sommairement paternalistes. Et, en général, la cause des droits de l’homme

\section{Ce qui a été entrepris depuis une vingtaine d'années}

\section{dans ce pays n'est pas négligeable du tout et est allé} dans le bon sens. les addictions, la violence, indépendamment de toute dimension migratoire au sens usuel du terme.

Dans ces conditions, je vois dans certaines critiques de «La migration comme métaphore» un risque de démotiver celles et ceux qui œuvrent dans le domaine et pourront se sentir épinglés - alors que la charge devrait être dirigée contre un modèle de société. L'important appareil documentaire du livre traite essentiellement de migration mais, à mon sens, cela n'invalide pas la pertinence de ma question. Et je regretterais que le fait non contesté que notre société pourrait être plus juste, globalement, discrédite indûment les actions médico-sociales et éducatives dans le champ de la migration.

\section{En guise de conclusion}

Le travail est académiquement et pratiquement substantiel, Métraux a une grande connaissance de la littérature et une grande expérience. Ce livre est bien écrit, l'auteur est une «plume» avec une vraie qualité littéraire, y compris par des trouvailles métaphoriques qui disent remarquablement ce qu'il entend dire.

Selon le préfacier Jean Furtos, l'ouvrage est destiné aux professionnels, aux politiques et aux honnêtes gens qui devraient comprendre le monde où nous vivons et ses processus migratoires permanents. J'ai dit ci-dessus des réserves. Peut-être convient-il se souvenir de la formule qui veut que les idéaux ne sont pas des objectifs que nous pouvons atteindre, mais ils nous servent de phares. Les buts de reconnaissance mutuelle et de co-construction avec les personnes migrantes d'un espace commun, dans une totale réciprocité/équité, au sein du pays d'accueil, sont des phares, mais il ne faudrait pas que le fait qu'ils ne sont pas rapidement atteints disqualifie ce qui est réalisé aujourd'hui et qui (imparfaitement) a sa valeur.

Un point majeur dans l'évaluation de la charge qu'instruit l'ouvrage est de savoir si ces critiques doivent être formulées spécifiquement pour ce qui est fait en matière de migration, ou si c'est une réalité générale qui a des conséquences dans ce domaine comme dans d'autres. Je penche pour la seconde interprétation. Il me parait (voir par exemple le Chapitre VII) que, en fait, l'auteur vise les multiples inégalités et inéquités qu'on constate et qui sont le reflet d'un modèle socio-politique. Il est vraisemblable que la gestion de la migration illustre des manques de la société suisse (ou européenne) en termes de justice sociale. Oui, on peut constater à propos de migrants des manifestations de discrimination, des attributions de déficits, leur non-implication dans les programmes mis en œuvre. Mais la même chose peut être dite pour d'autres groupes marqués par la précarité, la pauvreté,

\section{Références}

1 Goguikian Ratcliff B, Strasser O. Clinique de l'exil chroniques d'une pratique engagée. Genève: Georg Editeur; 2009. Analyse dans: Martin J. Les soins aux personnes migrantes, aux «sans voix» Nécessité d'une clinique transculturelle. Bioethica Forum. 2010;3(1): 29-31, et dans: Bull Méd Suisses. 2010;91(18):741-3.

2 Métraux JC. Deuils collectifs et création sociale. Paris: La Dispute; 2004.

3 Métraux JC. La migration comme métaphore. Paris: La Dispute; 2011.

4 Martin J. Interprétariat communautaire et soins aux personnes «invisibles».

Bull Méd Suisses. 2008;89(12/13):544. 